\title{
SSTR2 promoter hypermethylation is associated with the risk and progression of laryngeal squamous cell carcinoma in males
}

\author{
Zhisen Shen ${ }^{1 *}$, Xiaoying Chen ${ }^{2}$, Qun $\mathrm{Li}^{1,2}$, Chongchang Zhou ${ }^{1,2}$, Jinyun $\mathrm{Li}^{2}$, Huadan Ye ${ }^{2}$ and Shiwei Duan ${ }^{2 *}$
}

\begin{abstract}
Background: Somatostatin receptor 2 (SSTR2) encodes somatostatin receptor that can inhibit the cell proliferation of solid tumors. Promoter hypermethylation is likely to silence the expression of SSTR2. The goal of our study was to investigate the association between SSTR2 promoter methylation and the risk and progression of laryngeal carcinoma.

Methods: In the current study, tumor tissues and their adjacent non-tumor tissues were collected from a total of 87 laryngeal squamous cell carcinoma (LSCC) male patients. DNA methylation levels of nine SSTR2 promoter CpGs were measured using the bisulphite pyrosequencing technology.

Results: Our results revealed that there was a significantly increased SSTR2 promoter methylation in LSCC tissues than in their adjacent non-cancerous tissues (adjusted $P=0.003$ ). Breakdown analysis by age indicated that the significant association was mainly contributed by patients younger than 60 (adjusted $P=0.039$ ) but not in patients older than 60. Meanwhile, the significant association was observed in the patients with moderately (adjusted $P=0.037$ ) and well differentiated tissues (adjusted $P=0.028$ ), as well as the patients with histological stage IV (adjusted $P=0.031$ ). Multivariate Cox analysis suggested that SSTR2 promoter methylation was an independent prognostic factor of $\operatorname{LSCC}(\mathrm{HR}=1.127,95 \% \mathrm{Cl}=1.034-1.228)$.
\end{abstract}

Conclusions: In conclusion, SSTR2 promoter hypermethylation might be associated with the risk and progression of LSCC in males.

Keywords: Laryngeal carcinoma, SSTR2, DNA methylation, Male

\section{Background}

Laryngeal cancer is a devastating malignancy of head and neck, and its incidence and mortality rates are increasing recently [1]. Despite improvement of oncological and surgical treatments over the last 20 years, 5year survival rates of laryngeal cancer remained poor since the middle of 1980 s [2, 3]. More than $90 \%$ of laryngeal cancer has been pathologically identified as laryngeal squamous cell carcinoma (LSCC) [1]. According

\footnotetext{
* Correspondence: szs7216@163.com; duanshiwei@nbu.edu.cn Zhisen Shen and Xiaoying Chen are co-first authors of this work. ${ }^{1}$ Department of Otorhinolaryngology (Head and Neck Surgery), Lihuili Hospital of Ningbo University, Ningbo, Zhejiang 315040, China ${ }^{2}$ Zhejiang Provincial Key Laboratory of Pathophysiology, School of Medicine, Ningbo University, Ningbo, Zhejiang 315211, China
}

to the Cancer Facts \& Figures 2015 data, the majority of laryngeal cancer patients are males.

Currently, total laryngectomy and postoperative radiotherapy are the most common treatments for LSCC [4]. Due to serious impairment in laryngeal function and low quality life that brings for patients, the exact molecular pathogenesis of LSCC is still urgently needed to be explored. It is hypothesized that LSCC may result from the interactions of many complex factors, including environmental factors (smoking, alcohol consumption, air pollution, and infection) and genetic factors [5]. Meanwhile, accumulating studies suggest that there are numerous 
aberrant epigenetic modifications in laryngeal cancer [6].

Somatostatin (SST) is an important peptide in the regulation of cell secretion and proliferation [7]. It operates through engagement on a family of 5 transmembrane G-protein coupled somatostatin receptors (SSTRs 1-5) [8]. Somatostatins have a function of anti-proliferation, pro-apoptosis and anti-migration, and thus play a role in the suppression of tumor growth. Hypermethylation of SSTR1 gene along with reduced expression was found in head and neck squamous cell carcinoma [9], and the reversed SSTR5 methylation was shown to up-regulate SSTR5 mRNA expression in prostate cancer [10]. SSTR2 is widely distributed and it is responsible for the antiproliferative effect of somatostatin and its analogs in vitro and in vivo [11]. SSTR2 protein levels were significantly lower in the malignant larynx than the pre-malignant larynx [12]. However, there was a lack of association study between SSTR2 methylation and laryngeal cancer.

Epigenetic modifications are crucial for tumorigenesis [13]. In mammalian cells, DNA methylation, as one of the most common modifications, occurs mainly at the C5 position of cytosine-phosphateguanine $(\mathrm{CpG})$ dinucleotides [14]. Genes with aberrant DNA methylation have been shown with great potential in the early detection and prognosis of cancers [15-18]. In light of previous findings, we aimed to test whether SSTR2 promoter methylation contributed to the pathogenesis of laryngeal cancer.

\section{Methods}

\section{Tissues samples}

All the tumors and their paired adjacent non-tumor tissues were collected from 87 male patients ranging from 40 to 86 years old. All the tissues were postoperative laryngeal specimens obtained from the LSCC male patients. There were 73 smokers and 14 non-smokers. And there were 40 well differentiated cases (Figures 1a and $\mathrm{n}), 32$ moderately differentiated cases, and 15 poorly differentiated cases (Figures $1 \mathrm{c}$ and D) according to their pathologic diagnoses. And there were 25 stage I, 14 stage II, 10 stage III, and 38 stage IV patients. There were 43 cases aged younger than 60 years, 33 cases aged $60-70$ years, and 11 cases aged older than 70 years. Overall survival (OS) data was recorded from 54 out of 87 patients between June 2010 and August 2015. The controls were the non-cancerous adjacent tissues that were obtained from at least $5 \mathrm{~cm}$ outside the edge of tumors, although they might not accurately represent noncancerous control tissues. Generally, total laryngectomy is the most common treatment for laryngeal cancer patients. Moreover, surgery-only laryngeal cancer patients had significantly better survival than those with radiotherapy or chemotherapy [19]. In addition, chemoradiotherapy was shown to affect the DNA methylation level [20]. Therefore, we excluded the patients with radiotherapy or chemotherapy in our study. All the specimens were obtained fresh and stored at $-80{ }^{\circ} \mathrm{C}$. None of the patients had a history of hereditary cancer. All the participants in
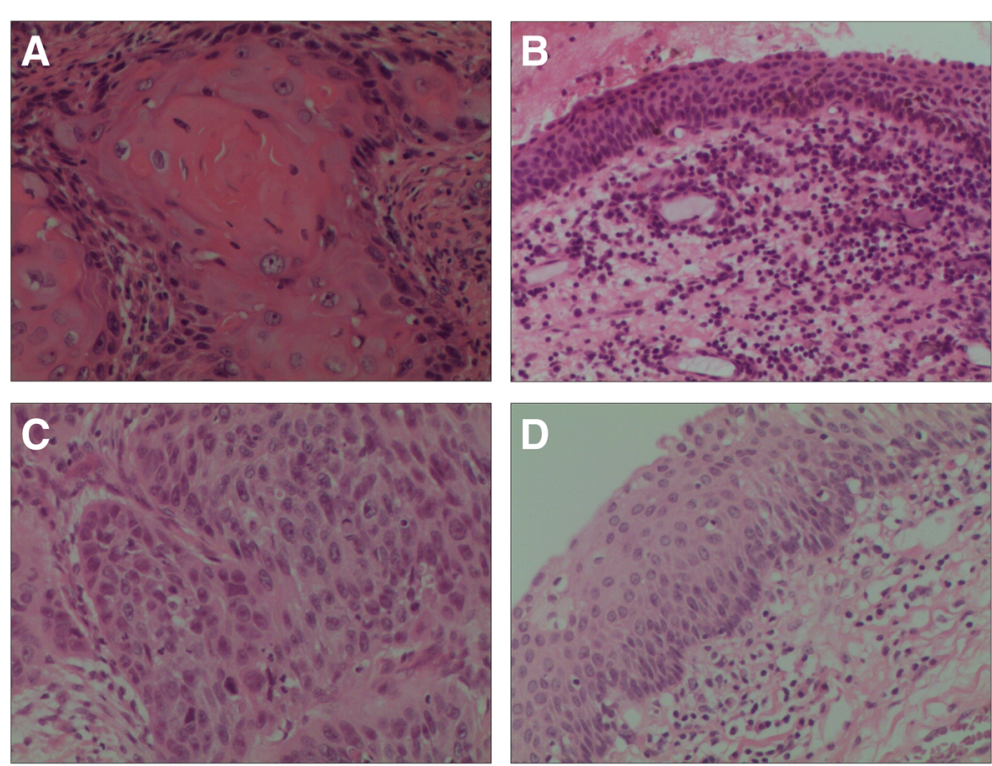

Fig. 1 Histopathology of test specimens. a Well differentiated tumor tissues of LSCC (x200). b Well differentiated non-tumor tissues of LSCC (x200). c Poorly differentiated tumor tissues of LSCC (x200). d Poorly differentiated non-tumor tissues of LSCC (x200) 
the study have signed the informed consent forms. Permission was also obtained from the local ethics committee to access the pathology archives at Department of Otolaryngology at Ningbo Lihuili Hospital, Ningbo, Zhejiang, China.

\section{Bisulfite pyrosequencing methylation assay and primer sequences}

Genomic DNA extraction from tumor samples and subsequent quantification procedures were as described before [21]. The details of DNA bisulfite conversion were as described previously [21]. The sequences were 5'AGGGTAGAGGAGTTAGGAATTT-3' for the forward primer, 5'-Biotin-ACCCCTCACCTTТАСТTTTC-3' for the reverse primer, and 5'-ACCCAACCACTATCCC-3' for the sequencing primer.

\section{Statistical analyses}

Statistical analyses were performed using SPSS v18.0 (SPSS Inc., Chicago, IL). Data was expressed as mean \pm standard deviation (SD). Analysis of variance (ANOVA) was used to evaluate the association between the risk factors (including age, smoking behavior, histological differentiation and clinical stage) and their relative methylation rate difference. Paired sample $T$-test and nonparametric test were performed to compare SSTR2 methylation levels between cancer tissues and adjacent tissues. All the $P$ values were adjusted by logistic regression. Multivariate Cox proportional hazard models were used to calculate hazard ratios (HR) and the corresponding $95 \%$ confidence intervals $(95 \% \mathrm{CI})$. A two-tailed $P$ $<0.05$ was considered to be significant. Figures were drawn using GraphPad Prism 6 software (GraphPad Inc., San Diego, CA).

\section{Results}

In the current study, we have tested nine CpG dinucleotides in the SSTR2 CpG island (CGI, chr17:71160923-
71162350) to measure SSTR2 methylation levels using the bisulfite pyrosequencing technology (Figure 2). The raw methylation data of nine CGs on SSTR2 gene promoter were presented in Supplemental Dataset 1. Using the mean methylation level, we preformed the association study between 87 male tumor tissues and their adjacent non-tumor tissues. In addition, the differences in SSTR2 methylation level were not statistically associated with age, smoking behavior, histological differentiation or clinical stage in tumor tissues or paired tissues (all adjusted $P>0.05$, data not shown).

Our results showed that SSTR2 promoter methylation levels in cancer tissues were significantly higher than in the paired non-tumor tissues (Figure 3a, $5.80 \pm 3.87 \%$ versus $3.67 \pm 3.22 \%, P=0.001$, adjusted $P=0.003)$. Subgroup analysis by the smoking status showed that SSTR2 promoter hypermethylation was associated with the risk of laryngeal cancer in the patients with and without smoking behaviors (Figure 3a, smokers: $5.46 \pm 4.94 \%$ versus $3.88 \pm 2.13 \%, P=0.013$, adjusted $P=0.021$; nonsmokers: $7.56 \pm 5.78$ versus $3.87 \pm 1.87, P=0.033$, adjusted $P=0.033)$. Further subgroup analysis by age showed that the patients aged $<60$ years old showed a statistically higher methylation in cancer tissues than in paired non-tumor tissues, but this association could not be found in those older than 60 years old (Figure 3b, $<60 \mathrm{y}$ : adjusted $P=0.039 ; 60 \mathrm{y} \sim$ : adjusted $P=0.059 ; 70 \mathrm{y} \sim$ : adjusted $P=0.287$ ).

DNA promoter methylation is associated with clinicopathological characteristics, such as differentiation and clinical stages $[22,23]$. Therefore, we conducted a histological differentiation-based comparison between tumor and non-tumor tissues. And our results showed a significant higher methylation in the well differentiated (Figure $3 C$, adjusted $P=0.028$ ) and moderately differentiated LSCC (adjusted $P=0.037$ ) compared with their adjacent tissues, but the significant association was not found in poorly differentiated LSCC compared with the controls

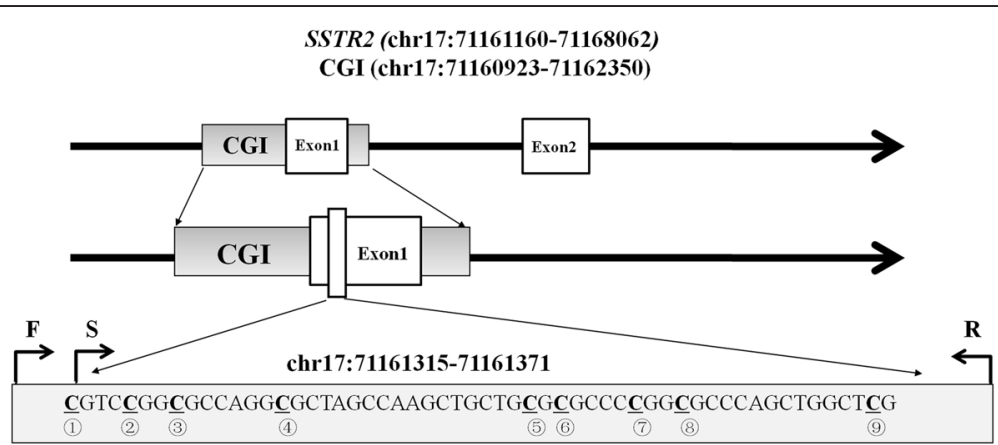

Fig. 2 The nine tested CpG dinucleotides in SSTR2 gene ${ }^{\text {a }}{ }^{\text {a }}$ : F stands for forward primer; $\mathrm{S}$ stands for sequencing primer; R stands for reverse primer 

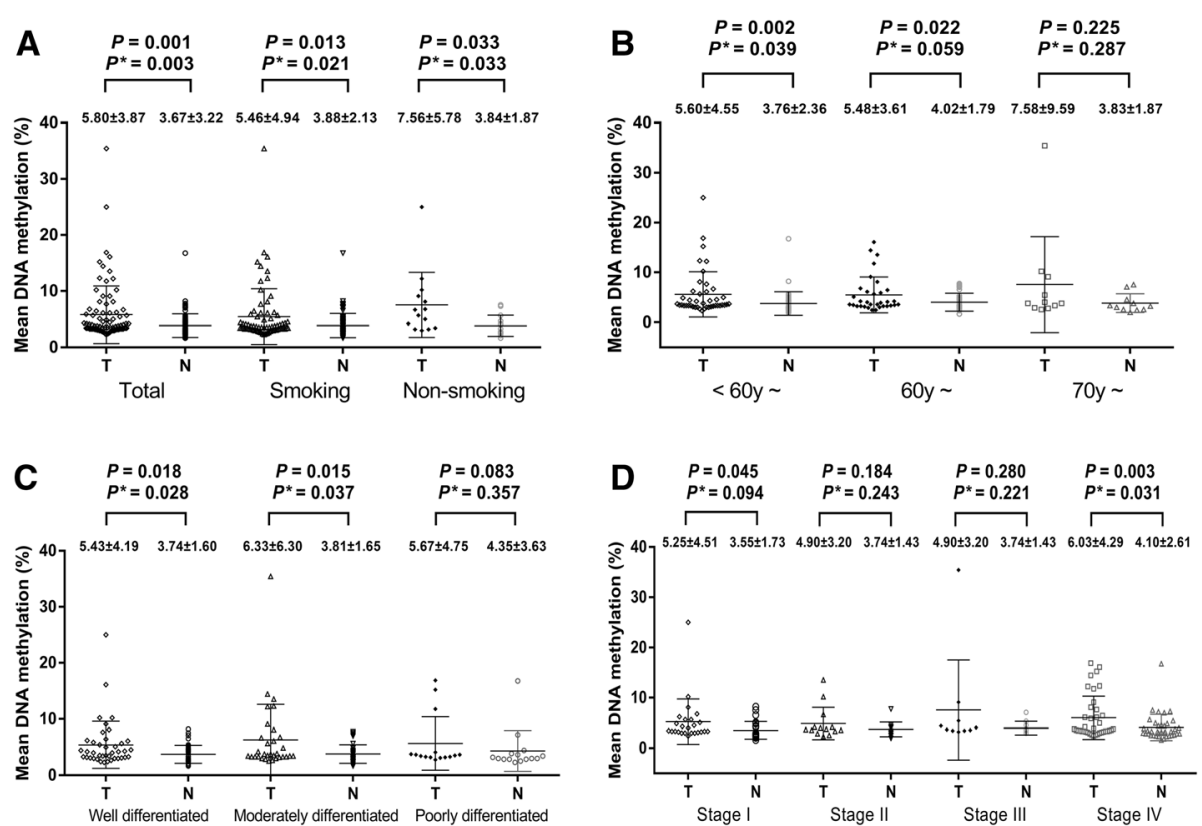

Fig. 3 Comparisons of mean methylation level of SSTR2 gene between tumor tissues and paired adjacent non-tumor tissues by (a) total and smoking behavior, (b) age, (c) differentiation and (d) clinical stage ${ }^{a}$. a: $T$ stands for tumor tissues; $\mathrm{N}$ stands for non-tumor tissues. $P^{*}$ : adjusted by age, smoking behavior, differentiation and clinical stage

(adjusted $P=0.357)$. In addition, clinical stage-based subgroup analysis showed a significant hypermethylation in stage IV (Figure 3D, adjusted $P=0.031$ ), but not in other stages (stage I: adjusted $P=0.094$; stage II: adjusted $P=0.243$; stage III: adjusted $P=0.221$ ).

In order to investigate the association between SSTR2 methylation level and OS in LSCC patients, we performed a multivariate Cox proportional hazard analysis by adjusting age, stage, differentiation and smoking behavior. As shown in Table 1, the poor OS of LSCC patients was found to be significantly associated with SSTR2 promoter hypermethylation $(\mathrm{HR}=1.127,95 \% \mathrm{CI}$ $=1.034-1.228)$.

\section{Discussion}

Our study firstly investigated the role of SSTR2 promoter methylation in the risk and progression of LSCC. A significantly increased SSTR2 promoter methylation was found in LSCC tissues than non-cancerous tissues. Meanwhile, multivariate Cox analysis suggested that SSTR2 promoter methylation was an independent prognostic factor of LSCC. Our results provided a clue for further studies on the role of SSTR2 in laryngeal carcinogenesis, and future studies were needed to confirm its potential as a biomarker for early diagnosis, therapy and prognosis of laryngeal cancer.

Previous laryngeal cancer study has shown that the overall pattern of SSTRs expression is with high levels of SSTR1, "loss" of SSTR2 and intermediate levels of
SSTR5 [24]. For other SSTR members, there was very little expression of SSTR3 detected in benign and premalignant specimens and malignant laryngeal tumors. Meanwhile, a variable degree of low to moderate levels of SSTR4 expression was detected across these three groups [12].

Table 1 Multivariate Cox proportional hazards analysis in 54 LSCC patients $^{\text {a }}$

\begin{tabular}{lllll}
\hline Characteristics & $\mathrm{N}$ & $P$ value & $\mathrm{HR}$ & $95 \% \mathrm{Cl}$ \\
\hline Age & 54 & 0.821 & 0.992 & $0.925-1.064$ \\
Stage & & & & \\
Stage I (Ref) & 15 & - & 1.000 & - \\
Stage II & 8 & 0.106 & 6.794 & $0.665-69.416$ \\
Stage III & 7 & 1.865 & 4.865 & $0.408-57.962$ \\
Stage IV & 24 & 0.016 & 13.630 & $1.636-113.539$ \\
Differentiation & & & & \\
Well (Ref) & 21 & - & 1.000 & - \\
Moderated & 21 & 0.706 & 1.267 & $0.370-4.344$ \\
Poorly & 12 & 0.555 & 1.401 & $0.457-4.299$ \\
Smoking behavior & & & & \\
No (Ref) & 7 & - & 1.000 & - \\
Yes & 47 & 0.271 & 2.699 & $0.461-15.813$ \\
SSTR2 methylation & 54 & 0.006 & 1.127 & $1.034-1.228$ \\
\hline
\end{tabular}

${ }^{a}$ : the overall survival information of 33 LSCC patients was unavailable; Ref: reference category; HR: hazard ratio 
DNA hypermethylation in the promoter of tumor suppressor genes (TSGs) inhibits transcriptional initiation and results in the silencing of TSGs. Hypermethylated promoters of tumor suppressor genes (CHD5 [25], CHFR [26], PTEN [27], FHIT [28], CDKN2B [29], APC [29], DAPK1 [29]) have been shown as an important epigenetic mechanism in LSCC. Moreover, accumulating studies have reported other aberrantly hypermethylated genes in LSCC, including CBY [30], IGFBP-rP1 [31], and MYCT1 [6]. Here, we provided another hypermethylated gene (SSTR2) in LSCC. Alternation of SSTR2 promoter methylation level in LSCC is not fully understood. Here, we observed a significantly increased SSTR2 promoter methylation in cancerous tissues than in their adjacent tissues, especially in the stage IV LSCC patients. Besides, we found that SSTR2 promoter hypermethylation could predict a poor OS of LSCC. Taken together, these supported that the "loss" of SSTR2 expression in laryngeal carcinoma, especially in the late stage, might be due to a higher methylation of SSTR2. Although mRNA levels were not determined in these specimens, our results tended to be compatible with the previous results of the previous laryngeal cancer study [32].

According to the Cancer Facts \& Figures 2015 data (http://www.cancer.org/research/cancerfactsstatistics/ cancerfactsfigures2015/index), most the laryngeal cancer patients are males. In the current study, we only recruited very few female samples. In order to get rid of heterogeneity by gender, we decided to focus on the study in males for the moment. Besides, we have performed smoking status, age, histological differentiation and stage subgroups analyses.

Age plays an important role in the carcinogenesis [33]. Interestingly, the significantly higher methylation was found in the younger population ( $<60$ years), which might provide a potentially age-specific biomarker of laryngeal cancer.

SSTR2 is widely expressed in normal tissues, and it is a negative regulator of cell proliferation in human tumorigenesis [34]. Increased SSTR2 expression led to strong up-regulation of cyclin-dependent kinase inhibitor p16, which then inhibited tumor cell cycle progression from $\mathrm{G} 1$ to $\mathrm{S}$ phase [35]. In addition, SSTR2 deficiency may contribute to the development of tissue invasion and metastasis process [36]. Lower mRNA levels of SSTR2 were expressed in the metastases from prostate cancers than in primary prostate cancers, meanwhile, decreased SSTR2 could predict poor prognosis of prostate cancer [35]. Our breakdown analysis showed a significant association of SSTR2 methylation with LSCC risk in moderately and well differentiated LSCC patients but not in the poorly differentiated patients. Additionally, there was a significant difference could be found in stage IV group, even adjusted by risk factors of age and smoking. Our results provided a hypothesis that the SSTR2 methylation might be involved in the metastasis and invasion of laryngeal cancer.

However, there were limitations in the current research to be considered. Firstly, our study was involved with 87 LSCC cancerous and 87 non-cancerous tissues. Power analysis showed $90.8 \%$ power for overall test and $15.1-100.0 \%$ power for the subgroup analyses by differentiation, histological stages of cancerous tissues and age. Thus, our observations in the total samples were reliable, however, the negative results in the subgroup analyses might need to be confirmed with larger sample size in the future. Secondly, the professional background was a potential risk factor in laryngeal disease. A previous study has reported that professional voice users such as singers may be susceptible to laryngeal disease [37]. Unfortunately, we didn't collect the professional background of patients.

\section{Conclusions}

This study indicated that SSTR2 promoter hypermethylation was associated with the risk and progression of laryngeal cancer in males. These findings provided clues for further studies on the role of SSTR2 in laryngeal carcinogenesis and its potential as a biomarker for early diagnosis, therapy and prognosis of laryngeal cancer.

\section{Abbreviations \\ CGI: CpG island; CpG: cytosine-phosphate-guanine; DNA: deoxyribonucleic acid; LSCC: laryngeal squamous cell carcinoma; LSCC: laryngeal squamous cell carcinoma; SSTR2: aomatostatin receptor 2.}

\section{Competing interests}

The authors declare that there is no conflict of interests regarding the publication of this article.

\section{Authors' contributions}

ZS and SD have contributed to the conception and design of the study. XY drafted the manuscript. QL, $J \mathrm{~L}$ and $H Y$ performed the collection and assembly of data, the analysis and interpretation of data, the statistical analysis. CZ participated in the design of the study, provided technical guidance and helped to draft the manuscript. All authors read and approved the final manuscript.

\section{Acknowledgments}

The research was supported by grants from Zhejiang Provincial Natural Science Foundation of China (No.LY14H160003), the Scientific Innovation Team Project of Ningbo (No.2012B2019), Ningbo Social Development Research Project (No.2012C5015), Ningbo Natural Science Foundation (No.2012A610208; 2012A610217; 2013A610217), Medical and Health Research Project of Zhejiang Province (No.2012ZDA042), Medical and Health Training Project of Zhejiang Province (No.2014PYA017).

Received: 4 November 2015 Accepted: 14 January 2016 Published online: 22 January 2016

\section{References}

1. Morshed K, Polz-Dacewicz M, Szymanski M, Polz D. Short-fragment PCR assay for highly sensitive broad-spectrum detection of human 
papillomaviruses in laryngeal squamous cell carcinoma and normal mucosa: clinico-pathological evaluation. Eur Arch Otorhinolaryngol. 2008;265 Suppl 1: S89-96.

2. Parkin DM, Bray F, Ferlay J, Pisani P. Estimating the world cancer burden: Globocan 2000. Int J Cancer. 2001;94:153-6.

3. Rudolph E, Dyckhoff G, Becher H, Dietz A, Ramroth H. Effects of tumour stage, comorbidity and therapy on survival of laryngeal cancer patients: a systematic review and a meta-analysis. Eur Arch Otorhinolaryngol. 2011;268:165-79.

4. Kada S, Hirano S, Tateya I, Kitamura M, Ishikawa S, Kanda T, et al. Ten years single institutional experience of treatment for advanced laryngeal cancer in Kyoto University. Acta Otolaryngol Suppl. 2010:68-73

5. Toporcov TN, Znaor A, Zhang ZF, Yu GP, Winn DM, Wei Q, et al. Risk factors for head and neck cancer in young adults: a pooled analysis in the INHANCE consortium. Int J Epidemiol. 2015;44:169-85.

6. Yang M, Li W, Liu YY, Fu S, Qiu GB, Sun KL, et al. Promoter hypermethylation-induced transcriptional down-regulation of the gene MYCT1 in laryngeal squamous cell carcinoma. BMC Cancer. 2012;12:219.

7. Guillermet-Guibert J, Lahlou H, Cordelier P, Bousquet C, Pyronnet S, Susini C. Physiology of somatostatin receptors. J Endocrinol Invest. 2005;28:5-9.

8. Goel A. Somatostatin methylation as a biomarker for gastric cancer: ready for "prime time" or for further validation? Dig Dis Sci. 2011;56:1-3.

9. Misawa K, Misawa Y, Kondo H, Mochizuki D, Imai A, Fukushima H, et al. Aberrant methylation inactivates somatostatin and somatostatin receptor type 1 in head and neck squamous cell carcinoma. PLoS One. 2015;10, e0118588.

10. Liu Z, Marquez M, Nilsson S, Holmberg AR. Incubation with somatostatin, 5-aza decitabine and trichostatin up-regulates somatostatin receptor expression in prostate cancer cells. Oncol Rep. 2008;20:151-4.

11. Buscail L, Esteve JP, Saint-Laurent N, Bertrand V, Reisine T, O'Carroll AM, et al. Inhibition of cell proliferation by the somatostatin analogue RC-160 is mediated by somatostatin receptor subtypes SSTR2 and SSTR5 through different mechanisms. Proc Natl Acad Sci U S A. 1995:92:1580-4.

12. Condon LT, Stafford ND, Bedford KJ, MacDonald AW, Atkin SL. The expression of somatostatin receptors 3,4 and 5 in laryngeal pathology. Eur Arch Otorhinolaryngol. 2008;265 Suppl 1:S63-7.

13. Torrisani J, Hanoun N, Laurell H, Lopez F, Maoret JJ, Souque A, et al. Identification of an upstream promoter of the human somatostatin receptor, hSSTR2, which is controlled by epigenetic modifications. Endocrinology. 2008;149:3137-47.

14. Xiaoying C, Huadan Y, Qingxiao H, Annan Z, Linlin T, Shiwei D. The effects of DNA methylation on the homeostasis in vascular diseases. Yi Chuan. 2015;37:221-32

15. Jiang $D$, Hong $Q$, Shen $Y, X u Y, Z$ Zhu $H$, Li Y, et al. The diagnostic value of DNA methylation in leukemia: a systematic review and meta-analysis. PLoS One. 2014;9, e96822

16. Huang T, Chen X, Hong Q, Deng Z, Ma H, Xin Y, et al. Meta-analyses of gene methylation and smoking behavior in non-small cell lung cancer patients. Sci Rep. 2015;5:8897.

17. Jiang D, Shen Y, Dai D, Xu Y, Xu C, Zhu H, et al. Meta-analyses of methylation markers for prostate cancer. Tumour Biol. 2014;35:10449-55.

18. Chen C, Wang L, Liao Q, Huang Y, Ye H, Chen F, et al. Hypermethylation of EDNRB promoter contributes to the risk of colorectal cancer. Diagn Pathol. 2013;8:199.

19. Li Z, Ding S, Zhong Q, Li G, Zhang Y, Huang XC. Significance of MMP11 and P14(ARF) expressions in clinical outcomes of patients with laryngeal cancer. Int J Clin Exp Med. 2015;8:15581-90.

20. Williamson JS, Harris DA, Beynon J, Jenkins GJ. Review of the development of DNA methylation as a marker of response to neoadjuvant therapy and outcomes in rectal cancer. Clin Epigenetics. 2015:7:70.

21. Xu L, Zheng D, Wang $L$, Jiang D, Liu H, Xu L, et al. GCK gene-body hypomethylation is associated with the risk of coronary heart disease. Biomed Res Int. 2014;2014:151723.

22. Zhang Y. Zhang J. Mol Biosyst: Identification of functionally methylated regions based on discriminant analysis through integrating methylation and gene expression data; 2015.

23. Chen AY, Fedewa S, Zhu J. Temporal trends in the treatment of early- and advanced-stage laryngeal cancer in the United States, 1985-2007. Arch Otolaryngol Head Neck Surg. 2011;137:1017-24.

24. Liu Y. Radiolabelled somatostatin analog therapy in prostate cancer: current status and future directions. Cancer Lett. 2006:239:21-6.
25. Wang J, Chen H, Fu S, Xu ZM, Sun KL, Fu WN. The involvement of CHD5 hypermethylation in laryngeal squamous cell carcinoma. Oral Oncol. 2011; 47:601-8

26. He L, Ji W, Yang J, Zhao X. Study of mRNA expression level and hypermethylation of CHFR promoter in the laryngeal squamous cell carcinoma tissue. Lin Chung Er Bi Yan Hou Tou Jing Wai Ke Za Zhi. 2010;24: 673-7.

27. Bai W, Li W, Chen $X$, Wang T. The relationship between hypermethylation of the PTEN promoter and laryngeal squamous cell carcinoma. Lin Chuang Er Bi Yan Hou Ke Za Zhi. 2006;20:254-6.

28. Yin D, Dong M. Methylation of promoter and expression of FHIT gene in laryngeal squamous cell carcinoma. Lin Chuang Er Bi Yan Hou Ke Za Zhi. 2005:19:253-5. 8.

29. Lopez F, Sampedro T, Llorente JL, Dominguez F, Hermsen M, Suarez C, et al. Utility of MS-MLPA in DNA methylation profiling in primary laryngeal squamous cell carcinoma. Oral Oncol. 2014;50:291-7.

30. Ren G, Zhao DA, Xu J, Li BA. Expression of CBY and methylation of CBY at promoter region in human laryngeal squamous cell carcinoma. Tumori. 2015;101:215-22.

31. Hu J, Zhao R, Wu G, Wang J. Expression and methylation status of IGFBP-rP1 gene in laryngocarcinoma. Lin Chung Er Bi Yan Hou Tou Jing Wai Ke Za Zhi. 2013:27:1352-4. 9

32. Stafford ND, Condon LT, Rogers MJ, MacDonald AW, Atkin SL. The expression of somatostatin receptors 1 and 2 in benign, pre-malignant and malignant laryngeal lesions. Clin Otolaryngol Allied Sci. 2003;28:314-9.

33. Chen K, Song F, He M, Li H, Qian B, Zhang W, et al. Trends in head and neck cancer incidence in Tianjin, China, between 1981 and 2002. Head Neck. 2009;31:175-82

34. Sakai K, Yonezawa T, Yamawaki H, Oyamada T. Alteration of somatostatin receptor 2 expression in canine mammary gland tumor. J Vet Med Sci. 2015:77:1319-22.

35. Hennigs JK, Muller J, Adam M, Spin JM, Riedel E, Graefen M, et al. Loss of somatostatin receptor subtype 2 in prostate cancer is linked to an aggressive cancer phenotype, high tumor cell proliferation and predicts early metastatic and biochemical relapse. PLoS One. 2014;9, e100469.

36. Shi WD, Meng ZQ, Chen Z, Lin JH, Zhou ZH, Liu LM. Identification of liver metastasis-related genes in a novel human pancreatic carcinoma cell mode by microarray analysis. Cancer Lett. 2009;283:84-91.

37. Vasilenko Iu S, Pavlikhin OG, Izgarysheva ZA. Analysis of the causes of vocal tract diseases in singers. Vestn Otorinolaringol. 2000:13-7.

\section{Submit your next manuscript to BioMed Central and we will help you at every step:}

- We accept pre-submission inquiries

- Our selector tool helps you to find the most relevant journal

- We provide round the clock customer support

- Convenient online submission

- Thorough peer review

- Inclusion in PubMed and all major indexing services

- Maximum visibility for your research

Submit your manuscript at www.biomedcentral.com/submit 